\title{
Towards Plasmon-Exciton Hybridization at the Nanoscale using STEM EELS
}

Andrew B. Yankovich ${ }^{1 *}$, Battulga Munkhbat ${ }^{1}$, Denis G. Baranov ${ }^{1}$, Jorge Cuadra ${ }^{1}$, Erik Olsén ${ }^{1}$, Hugo Lourenço-Martins' ${ }^{2}$, Luiz H. G. Tizei ${ }^{2}$, Mathieu Kociak², Eva Olsson' ${ }^{1}$, Timur Shegai ${ }^{1}$

1. Department of Physics, Chalmers University of Technology, Gothenburg, Sweden.

2. Laboratoire de Physique des Solides, Univ. Paris-Sud, Orsay, France.

* Corresponding author: andrew.yankovich@chalmers.se

Enhancing our control over the interactions between light and matter at the smallest possible length scales will help realize next-generation photonics-based applications. In the regime of strong coupling, light and matter can hybridize into new states called polaritons. Historically, hybridized polaritons have been studied using optical microcavities coupled to quantum emitters, such as atoms or quantum wells, which have led to several remarkable breakthroughs. The strength of the interaction is determined by comparing the Rabi frequency (the rate of coherent energy exchange between the optical cavity and quantum emitter) to the decay rates of the cavity and emitter. Weak coupling occurs when the Rabi frequency is slower than the mode decay rates, resulting in spontaneous photon emission. Strong coupling occurs when the Rabi frequency exceeds the decay rates, resulting in Rabi splitting and the genesis of two new coupled polaritons. These polaritons exhibit inseparable light and matter properties that modify the systems properties. Recently, plasmon-exciton polaritons (PEPs) have been realized using hybrid systems composed of localized surface plasmons (LSPs) in nanoparticles (NPs) and excitons in 2D transition metal dichalcogenide (TMDC) materials, extending polaritonic concepts to ultracompact footprints and room temperature operation [1].

Optical scattering spectroscopy has allowed for significant understanding of PEPs, but imaging their sub-wavelength nature is still an open problem because of the diffraction-limited spatial resolution. STEM EELS has unprecedented spatial resolution and has enabled various discoveries about the basic nature of LSPs and excitons. However, STEM EELS has not yet been utilized to detect PEPs, even though the possibility has been recently theoretically predicted [2].

Here, we present a STEM EELS study on hybrid plasmon-exciton systems composed of Ag NPs and few-layer $\mathrm{WS}_{2}$. STEM experiments were performed on a FEI Titan 80-300 microscope equipped with a Schottky field emission gun, Wien type monochromator, probe aberration corrector, and Gatan Imaging Filter (GIF) Tridiem 866 spectrometer. Monochromated STEM EELS experiments were performed at 80 $\mathrm{keV}$ with $\sim 1 \mathrm{~nm}$ spatial resolution and 40-70 meV energy resolution. The monochromator was operated in accelerating mode, which allowed for significantly improved energy resolution compared to the normal monochromator settings. Specific techniques were used to improve data quality and allowed for resolving the extremely small signals from these plasmon-exciton systems. This included acquiring EELS data using fast acquisition point spectral series and spectrum images, followed by processing the data using manual dark spectrum subtraction, energy instability correction, removal of spikes, and Richardson-Lucy deconvolution [3].

The wet-chemically synthesized Ag NPs have a triangular shape, single crystalline structure, $70-110 \mathrm{~nm}$ side lengths, clear faceted surfaces, and sharp 5-10 nm radius corners (Fig. 1a-b). Correlative optical DF and EELS experiments (Fig. 1c) reveal that the uncoupled NPs host bright dipolar LSPs that are most strongly excited using an electron beam positioned at the NP corners. The $\mathrm{WS}_{2}$ flakes host Wannier- 
Mott excitons that have more than one order of magnitude smaller EELS excitation probability compared to the dipolar LSP. Despite this, they are visible in experiments (Fig. 1c) because we use high signal-to-noise ratio techniques. The $\mathrm{WS}_{2} \mathrm{~A}-, \mathrm{B}-$, and C- exciton peaks are observed in EELS at $~ 2.0$, $\sim 2.4 \mathrm{eV}$ and $\sim 2.75 \mathrm{eV}$, respectively. To create the hybrid system, the Ag NP size must be chosen so that the dipolar LSP spectrally overlaps with the $\mathrm{WS}_{2}$ A-exciton, enabling hybridization.

To predict and interpret experimental EELS signals from the uncoupled and coupled systems, we simulate EELS spectra using the MNPBEM toolbox [4]. The experimental EELS observations of the uncoupled LSPs and excitons are in good agreement with the simulated predictions. Additionally, EELS simulations of coupled systems are used to predict at what $\mathrm{WS}_{2}$ thickness and EELS energy resolution the PEP mode splitting is resolvable (Fig. 2a-c).

In this presentation, we will show our PEP results that correlate EELS, optical DF, morphological analysis and numerical optical and EELS simulations. Our findings open up the possibility for in-depth studies of plasmon-exciton interactions with unprecedented spatial resolution [5].

References:

[1] M Stührenberg et al., Nano Letters 18 (2018), p. 5938.

[2] A Konečná et al., ACS Nano 12 (2018), p. 4775.

[3] AB Yankovich et al., ACS Nano 11 (2017), p. 4265.

[4] U Hohenester and A Trügler, Comput. Phys. Commun. 183 (2012), p. 370.

[5] We acknowledge funding from the Swedish Research Council (VR), the Engkvist Foundation for financial support, and the European Union's Horizon 2020 research and innovation program under grant agreement No 823717 - ESTEEM3.

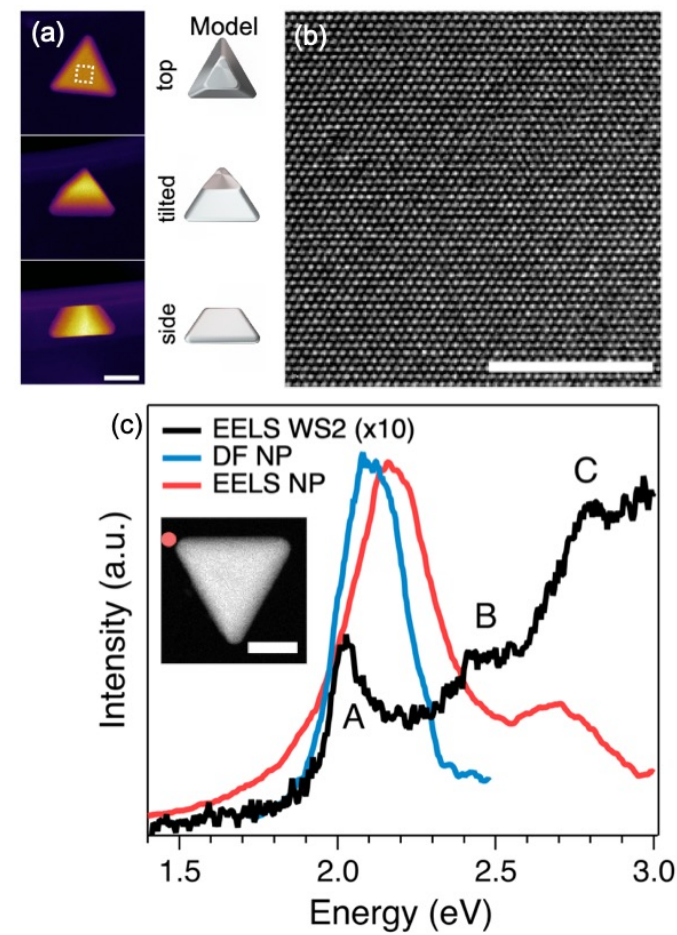

Figure 1. (a) HAADF STEM tilt series images of the same NP and a model structure. (b) HRTEM image of a NP, such as from the white box in (a). (c) Correlative EELS and optical DF scattering spectra from an uncoupled NP (inset HAADF) and $\mathrm{WS}_{2}$. Scale bars are $(\mathrm{a}, \mathrm{b}, \mathrm{c}) 50,5,50 \mathrm{~nm}$.

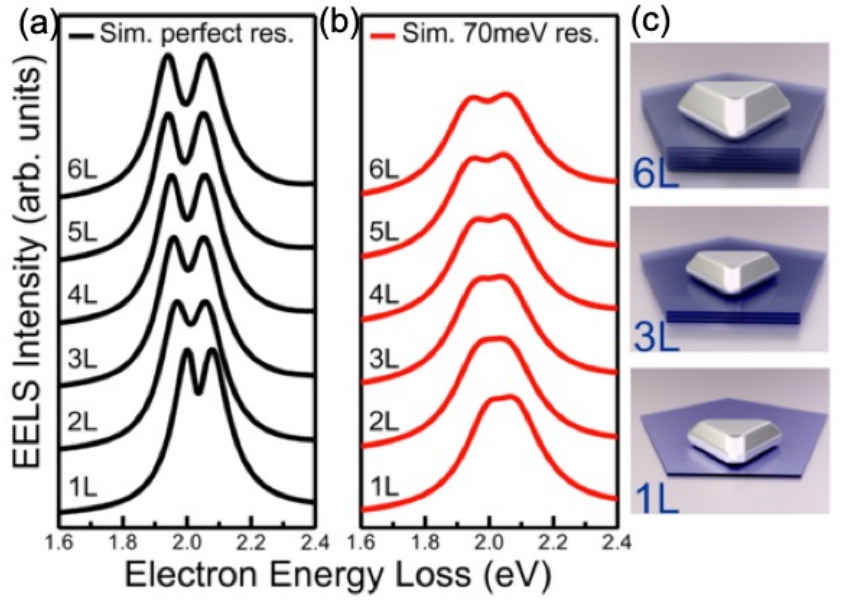

Figure 2. Simulated EELS spectra of hybrid Ag NP $-\mathrm{WS}_{2}$ systems for models with a $\mathrm{WS}_{2}$ thickness between 1 and 6 layers using (a) no spectral broadening and (b) $70 \mathrm{meV}$ spectral broadening. Mode splitting is predicted for all thicknesses in (a), but only for $>3$ layers in (b). (c) Schematic figures of the 1, 3, and 6 layer models. 